\title{
Factores demográficos y signos clínicos de pacientes con trauma penetrante abdominal en Yucatán
}

\author{
Demographic factors and clinical signs of patients \\ with penetrating abdominal injury in Yucatán \\ Sergio Isidro Gamboa Hoil, * María Fidelia Cárdenas Marrufo**
}

\footnotetext{
Palabras clave: Trauma abdominal penetrante, signos clínicos, arma blanca, laparotomía terapéutica,

laparotomía no terapéutica.

Key words: Penetrating abdominal injury, clinical signs, stab wounds, therapeutic laparotomy, non-therapeutic laparotomy.
}

* Cirugía General, Hospital General Agustín O'Horán, Secretaría de Salud de Yucatán; Mérida, Yucatán, México. ** Unidad Interinstitucional de Investigación Clínica y Epidemiológica, Facultad de Medicina, Universidad Autónoma de Yucatán; Mérida, Yucatán, México.

\section{RESUMEN}

Antecedentes: La tasa de trauma penetrante de abdomen (TPA) con arma blanca en el estado de Yucatán es de 57\%; es una de las principales causas de morbilidad y la principal causa de discapacidad en personas en edad productiva. Hasta la fecha se han descrito laparotomías no terapéuticas en un $40 \%$. Es difícil considerar alguna herramienta única para descartar una conducta quirúrgica; ante ello, ha aumentado el interés en identificar las características de los pacientes y el papel de los signos clínicos para establecer el tratamiento de esta patología. Objetivo: Conocer los factores demográficos y signos clínicos de los pacientes con trauma penetrante abdominal ingresados en el Hospital Agustín O’Horán del estado de Yucatán. Material y métodos: Estudio retrospectivo de 105 pacientes con trauma penetrante en el abdomen anterior intervenidos quirúrgicamente en un lapso de cinco años (de enero de 2010 a diciembre de 2014) en el Hospital General Agustín O’Horán. Se compararon las laparotomías terapéuticas y las no terapéuticas. Se analizó la edad, género, estado civil, residencia, índice de masa corporal, causa de la lesión, número de lesiones, área y órgano lesionados, y los signos vitales. Resultados: De 105 pacientes, 99 fueron hombres (94\%), solteros, con edad promedio de 31.3 años, que habitaban en el interior del estado. La causa más frecuente fue agresión por terceras personas con arma punzocortante en el epigastrio, con una única herida, asociada al consumo de alcohol. Resultaron con laparotomía no terapéutica 41 pacientes $(39 \%)$. Encontramos asociación estadística ( $\mathrm{p}<$ 0.05 ) en cuanto al lugar de residencia y en los signos vitales (frecuencia cardiaca mayor a 80 latidos por minuto, frecuencia respiratoria mayor a 16 respiraciones por minuto), irritación peritoneal y el resultado de laparotomías terapéuticas. Conclusión: En pacientes que presentan heridas en el abdomen anterior, en condiciones estables y asintomáticos, no se puede descartar un manejo quirúrgico; sin embargo, la inestabilidad hemodinámica y la irritación peritoneal son factores para considerar un procedimiento terapéutico.

\section{ABSTRACT}

Background: The rate of penetrating abdominal injury (PAI) with stab wounds in the state of Yucatan is 57\%; it is the leading cause of morbidity and disability in people in productive age. To date, nontherapeutic laparotomies have been described in $40 \%$. It is difficult to consider a unique tool to predict the need for laparotomies, which has increased the interest to determine the characteristics of patients and the role of the clinical signs to establish the treatment of this pathology. Objective: To determine the demographic factors and clinical signs of penetrating abdominal injuries in patients admitted in the General Hospital Agustin O'Horán of the state of Yucatán. Material and methods: This is a retrospective study of 105 records of patients with penetrating anterior abdomen injuries who underwent surgery in a five-year period (January 2010 to December 2014) in the General Hospital Agustín O'Horán. Therapeutic and nontherapeutic laparotomies were compared. Gender, marital status, residence, body mass index, cause of injury, number of injuries, injured body area, age and clinical signs were analyzed. Results: Of 105 patients, 99 were male (94\%), single, with an average age of 31.3 years, who lived in the interior of the state. The most frequent cause was personal violence, with a knife, in the epigastric zone, with a single wound associated with alcohol abuse. Forty-one patients (39\%) had nontherapeutic laparotomy. We found a statistical association $(p<0.05)$ regarding the place of residence, the clinical signs (heart rate higher than 80 beats per minute, breathing rate greater than 16 breaths per minute), peritonitis, and the result of therapeutic laparotomies. Conclusion: Surgical management cannot be discarded in patients with penetrating abdominal injury in asymptomatic and hemodynamic stability conditions; however hemodynamic instability and peritonitis are factors that should be considered for a therapeutic procedure.

\section{ANTECEDENTES}

$\mathrm{E}^{\mathrm{n}}$ n México, la tasa de trauma penetrante en abdomen (TPA) por arma blanca es de
$63.3 \%$, y en Yucatán 57\%, con una mortalidad de $2 \%$; es la quinta causa de morbilidad y la principal causa de discapacidad en personas en edad productiva. ${ }^{1,2}$ 
Es difícil considerar algún método o herramienta diagnóstica única para predecir la necesidad de laparotomía. ${ }^{3}$ Desde el año 1960 , Shaftan observó que en laparotomías obligadas por TPA, $40 \%$ resultaban no terapéuticas, con una morbilidad de entre tres y $16 \%$, y una mortalidad de $1 \%{ }^{4}$

La laparotomía exploradora es considerada imperativa en pacientes que presentan inestabilidad hemodinámica e irritación abdominal, estén bajo efectos de sustancias como drogas o alcohol, y con otras lesiones asociadas como fractura, trauma craneoencefálico y/o medular. ${ }^{5}$

La limitación o carencia de instrumentos diagnósticos en hospitales de nuestro país hace evidente el difícil abordaje de los pacientes en duda diagnóstica; es entonces donde las características de la lesión y la clínica se convierten en indispensables en la evaluación de los pacientes con trauma, en especial el TPA. ${ }^{6}$

\section{Objetivo}

Conocer los factores demográficos y signos clínicos de los pacientes con trauma penetrante abdominal ingresados en el Hospital General Agustín O'Horán del Estado de Yucatán.

\section{MATERIAL Y MÉTODOS}

Nuestro diseño fue observacional, analítico, retrospectivo, transversal. El muestreo fue no aleatorio por conveniencia. Se incluyeron todos los expedientes de pacientes con herida penetrante en el abdomen anterior ingresados e intervenidos quirúrgicamente en un periodo de cinco años (de enero de 2010 a diciembre de 2014) en el Hospital General Agustín O'Horán del Estado de Yucatán. Se excluyeron los pacientes menores de 15 años, las embarazadas, aquellos con antecedente de hipertensión arterial diagnosticada, heridas múltiples que comprometieran la vida y las ocasionadas por arma de fuego. Se eliminaron los expedientes cuya información estaba incompleta. Se utilizó el programa SPSS 19.0. Se usó la estadística descriptiva para las frecuencias y porcentajes de las variables. Se empleó el análisis univariado para las variables categóricas y no paramétricas mediante tablas de contingencia de $2 \times 2$ (Ji al cuadrado). Los resultados con $\mathrm{p} \leq 0.05$ fueron considerados significativos.

Con base en la Declaración de Helsinki y la Ley General de Salud en Materia de Investigación, artículo 17, el presente estudio cae en la categoría de sin riesgo; por lo tanto, no se solicitó consentimiento informado a los pacientes. La información se manejó de manera confidencial.

\section{RESULTADOS}

En el periodo comprendido en el estudio se obtuvieron 112 expedientes; sin embargo, de éstos, siete no cumplieron los criterios de inclusión, por lo que quedaron un total de 105.

Con respecto a los factores demográficos de la población, encontramos que 99 eran del

\begin{tabular}{|c|c|c|c|c|c|}
\hline Variable & Escala de medición & $\begin{array}{c}\text { Laparotomía } \\
\text { no terapéutica (\%) }\end{array}$ & $\begin{array}{l}\text { Laparotomía } \\
\text { terapéutica }(\%)\end{array}$ & Total (\%) & $\mathrm{p}$ \\
\hline \multirow[t]{3}{*}{ Género } & Masculino & $38(36.1)$ & $61(58)$ & 99 (94.2) & \multirow{3}{*}{0.5} \\
\hline & Femenino & $3(2.8)$ & $3(2.8)$ & $6(5.7)$ & \\
\hline & Total & $41(39)$ & $64(60.9)$ & $105(100)$ & \\
\hline \multirow[t]{3}{*}{ Estado civil } & Casado & $14(13.3)$ & $13(12.3)$ & $27(25.7)$ & \multirow{3}{*}{0.16} \\
\hline & Soltero & $20(19.0)$ & $39(37.1)$ & $59(56.1)$ & \\
\hline & Unión libre & $7(6.6)$ & $12(11.4)$ & $19(18.0)$ & \\
\hline \multirow[t]{2}{*}{ Residencia } & Mérida & $22(20.9)$ & $22(20.9)$ & 44 (41.9) & \multirow[t]{2}{*}{0.05} \\
\hline & Interior del estado & $19(18.0)$ & $42(40)$ & $61(58)$ & \\
\hline \multirow{3}{*}{$\begin{array}{l}\text { Índice de masa } \\
\text { corporal }\end{array}$} & Bajo & $17(16.1)$ & 23 (21.9) & $40(38.0)$ & \multirow{3}{*}{0.8} \\
\hline & Normal & $18(17.1)$ & $31(29.5)$ & 49 (46.6) & \\
\hline & Alto & $6(5.7)$ & $10(9.5)$ & $16(15.2)$ & \\
\hline
\end{tabular}


género masculino (94\%), con una relación $\mathrm{H}: \mathrm{M}$ 16:1 y un promedio de 31.3 años (Cuadro I).

La hora más frecuente de ingreso fue a las 8 p. m., en un $12 \%$, seguida de la 1 a. m., con $11 \%$. El tiempo de resolución quirúrgica tras su ingreso fue mínimo de dos horas y máximo de 24 horas.

Las lesiones ocasionadas por terceras personas fueron $32.5 \%$. La frecuencia del arma blanca utilizada y el resultado de la laparotomía realizada se observan en el cuadro II, donde también se exponen las frecuencias de TPA según el área abdominal afectada.

Resultaron con laparotomía no terapéutica 41 pacientes (39\%). Los hallazgos de las laparo- tomías se describen en el cuadro III. Los signos clínicos y la exploración física fueron valorados por el personal que brindó la atención en el Servicio de Urgencias. Los signos vitales fueron analizados al ingreso y posteriormente a la reanimación básica en el Servicio de Urgencias. Los datos se pueden ver en los cuadros $I V$ y $V$. Los resultados del análisis de la exploración física se observan en el cuadro VI.

La hemorragia promedio fue $200 \mathrm{~cm}^{3}$; los días de hospitalización promedio fueron seis. Dos pacientes se intervinieron, uno por evisceración en un paciente con trastorno psiquiátrico y otro por lesión inadvertida de víscera hueca.

\begin{tabular}{|c|c|c|c|c|c|}
\hline Arma blanca & $\begin{array}{c}\text { Laparotomía } \\
\text { no terapéutica }(\%)\end{array}$ & $\begin{array}{c}\text { Laparotomía } \\
\text { terapéutica (\%) }\end{array}$ & & $\begin{array}{c}22 \\
(20.9 \%)\end{array}$ & $(13.3 \%)$ \\
\hline $\begin{array}{l}\text { Cuchillo } \\
\text { Navaja }\end{array}$ & $\begin{array}{l}5(4.7) \\
3(2.8)\end{array}$ & $\begin{array}{l}4(3.8) \\
2(1.9)\end{array}$ & $\begin{array}{c}6 \\
(5.7 \%) \\
\end{array}$ & $\begin{array}{c}20 \\
(19.0 \%) \\
\end{array}$ & $\begin{array}{c}18 \\
(17.1 \%)\end{array}$ \\
\hline Desarmador & 0 & $1(0.9)$ & & 0 & 6 \\
\hline Machete & 0 & $1(0.9)$ & & $(0 \%)$ & \\
\hline Sin especificar & $33(31.4)$ & $56(53.3)$ & & Anterior & \\
\hline
\end{tabular}

\begin{tabular}{|c|c|c|c|c|c|c|}
\hline \multirow[b]{3}{*}{ Órgano lesionado } & \multicolumn{6}{|c|}{$\begin{array}{c}\text { Cuadro III. Frecuencia de órganos lesionados y el tratamiento } \\
\text { que se les realizó según resultado de la laparotomía. }\end{array}$} \\
\hline & \multicolumn{6}{|c|}{ Procedimiento realizado } \\
\hline & $\begin{array}{l}\text { Ninguno } \\
(\%)\end{array}$ & $\begin{array}{c}\text { Cierre } \\
\text { primario }(\%)\end{array}$ & $\begin{array}{l}\text { Anastomosis } \\
\quad(\%)\end{array}$ & $\begin{array}{l}\text { Se quitó el } \\
\text { órgano (\%) }\end{array}$ & $\begin{array}{l}\text { Se cauterizó } \\
\quad(\%)\end{array}$ & Total $(\%)$ \\
\hline Hígado & $6(5.7)$ & $1(0.9)$ & $0(0)$ & $0(0)$ & $5(4.7)$ & $12(11.4)$ \\
\hline Bazo & $0(0)$ & $0(0)$ & $0(0)$ & $2(1.9)$ & $0(0)$ & $2(1.9)$ \\
\hline Estómago & $0(0)$ & $8(7.6)$ & $0(0)$ & $0(0)$ & $0(0)$ & $8(8.6)$ \\
\hline Intestino delgado & $0(0)$ & $25(23.8)$ & $2(1.9)$ & $0(0)$ & $0(0)$ & $27(25.7)$ \\
\hline Intestino grueso & $0(0)$ & $16(15.2)$ & $0(0)$ & $1(0.9)$ & $0(0)$ & $17(16.2)$ \\
\hline Epiplón & $5(4.7)$ & $0(0)$ & $0(0)$ & $0(0)$ & $0(0)$ & $5(4.8)$ \\
\hline Pared & $23(21.9)$ & $0(0)$ & $0(0)$ & $0(0)$ & $1(0.9)$ & $24(22.9)$ \\
\hline Meso & $2(1.9)$ & $0(0)$ & $0(0)$ & $0(0)$ & $1(0.9)$ & $3(2.9)$ \\
\hline No penetró & $5(4.7)$ & $0(0)$ & $0(0)$ & $0(0)$ & $0(0)$ & $5(4.8)$ \\
\hline Riñón & $0(0)$ & $0(0)$ & $0(0)$ & $2(1.9)$ & $0(0)$ & $2(1.9)$ \\
\hline \multicolumn{7}{|c|}{ Según resultado de las laparotomías } \\
\hline No terapéutica & $41(39)$ & $0(0)$ & $0(0)$ & $0(0)$ & $0(0)$ & $41(39)$ \\
\hline Terapéutica & $0(0)$ & $50(47.6)$ & $2(1.9)$ & $5(4.8)$ & $7(6.7)$ & $64(60.9)$ \\
\hline Total & $41(39)$ & $50(47.6)$ & $2(1.9)$ & $5(4.8)$ & $7(6.7)$ & $105(100)$ \\
\hline
\end{tabular}




\section{DISCUSIÓN}

Encontramos que el trauma penetrante de abdomen anterior fue más frecuente en el sexo masculino, en un $94 \%$, con una relación $\mathrm{H}: \mathrm{M}$ 16.5:1, similar a lo observado en la literatura nacional por Pinedo (en 2006) de 93.6\%, Castillo (en 2011) de 96.1\% y Díaz (en 2012) de 94.4\%, con una relación H:M desde 14:8 hasta 25:1. Fue más frecuente en la cuarta década de la vida con respecto a la tercera, según lo publicado por Pinedo, Castillo y Díaz. 1,7,8 Observamos mayor frecuencia en solteros (56\%), mientras que Pinedo encontró que fue más común en casados (52\%). Aunque se ha descrito que en pacientes obesos la energía cinética de la agresión dismi- nuye y con ello se evita que el arma penetre al abdomen, ${ }^{1}$ en nuestro estudio no hallamos una relación entre el índice de masa corporal (IMC) y un resultado negativo en la laparotomía, aunque es importante destacar que la población con IMC mayor a 25 fue sólo de un 15\%.

El periodo en que se observó la mayor frecuencia de lesiones fue entre las 8 p. m. y las 2 a. m., con un $34 \%$, similar a lo descrito por Pinedo (58.23\%) y Castillo (50\%). ${ }^{1,7}$ El consumo de alcohol estuvo presente en el $98 \%$ de los TPA, lo que fue superior a lo encontrado por Romero en el estado de Yucatán hace más de dos décadas (58.5\%). ${ }^{9}$ La violencia interpersonal fue la etiología más frecuente, en $88.5 \%$, similar a lo publicado por Pinedo $(86 \%) ;{ }^{1}$ el

\begin{tabular}{lccccc}
\hline \multicolumn{5}{c}{$\begin{array}{c}\text { Cuadro IV. Frecuencia de los signos vitales y su relación con el resultado } \\
\text { de la laparotomía a su ingreso al Servicio de Urgencias. }\end{array}$} \\
\cline { 2 - 4 } & \multicolumn{5}{c}{ Signos vitales a su ingreso } \\
Variable & Laparotomía no & Laparotomía \\
& Escala de medición & $\begin{array}{c}\text { Lerapéutica (\%) } \\
\text { terapéutica (\%) }\end{array}$ & Total (\%) & p \\
\hline \multirow{2}{*}{ Tensión arterial } & Hipotenso & $4(3.8)$ & $11(10.4)$ & $15(14.2)$ & \\
& Normotenso & $31(29.5)$ & $46(43.8)$ & $77(73.3)$ & 0.52 \\
Frecuencia cardiaca & Hipertenso & $6(5.7)$ & $7(6.6)$ & $13(12.3)$ & \\
& Bradicardia & $1(0.9)$ & $2(1.9)$ & $3(2.8)$ & \\
& Normal & $22(20.9)$ & $16(15.2)$ & $38(36.1)$ & 0.01 \\
Frecuencia respiratoria & Taquicardia & $18(17.1)$ & $46(43.8)$ & $64(60.9)$ & \\
& Normal & $13(12.3)$ & $9(8.5)$ & $22(20.9)$ & 0.03 \\
& Taquipnea & $28(26.6)$ & $55(52.3)$ & $83(79.0)$ &
\end{tabular}

\begin{tabular}{|c|c|c|c|c|c|}
\hline \multirow[b]{2}{*}{ Variable } & \multicolumn{4}{|c|}{ Signos vitales posteriores a la reanimación básica } & \multirow[b]{2}{*}{$\mathrm{p}$} \\
\hline & Escala de medición & No terapéutica (\%) & Terapéutica (\%) & Total (\%) & \\
\hline \multirow[t]{3}{*}{ Tensión arterial } & Hipotenso & $0(0)$ & $5(4.7)$ & $5(4.7)$ & \multirow{3}{*}{0.1} \\
\hline & Normotenso & $27(25.7)$ & $43(40.9)$ & $70(66.6)$ & \\
\hline & Hipertenso & 14 (13.3) & $16(15.2)$ & $30(28.5)$ & \\
\hline \multirow[t]{3}{*}{ Frecuencia cardiaca } & Bradicardia & $2(1.9)$ & $2(1.9)$ & $4(3.8)$ & \multirow{3}{*}{0.9} \\
\hline & Normal & $26(24.7)$ & $31(29.5)$ & $57(54.2)$ & \\
\hline & Taquicardia & $13(12.3)$ & $31(29.5)$ & 44 (41.9) & \\
\hline \multirow[t]{2}{*}{ Frecuencia respiratoria } & Normal & $20(19)$ & $29(27.6)$ & 49 (46.6) & \\
\hline & Taquipnea & $21(20)$ & $35(33.3)$ & $56(53.3)$ & 0.7 \\
\hline
\end{tabular}




\begin{tabular}{lccccc}
\hline \multicolumn{5}{c}{$\begin{array}{c}\text { Cuadro VI. Frecuencia de los signos clínicos y su relación con el resultado } \\
\text { de la laparotomía a su ingreso al Servicio de Urgencias. }\end{array}$} \\
\hline Variable & Escala de medición (\%) & No terapéutica (\%) & Terapéutica (\%) & Total (\%) & p \\
\hline Peristalsis & Sí & $30(28.5)$ & $29(27.6)$ & $59(56.1)$ & \\
& No & $3(2.8)$ & $5(4.7)$ & $8(7.6)$ & 0.4 \\
Irritación peritoneal & No específica & $8(7.6)$ & $30(28.5)$ & $30(28.5)$ & \\
& Sí & $0(0)$ & $14(13.3)$ & $14(13.3)$ & \\
& No & $24(22.8)$ & $19(18)$ & $43(40.9)$ & 0.0001 \\
Exposición de epiplón & No específica & $17(16.1)$ & $31(29.5)$ & $48(45.7)$ & \\
& Sí & $9(8.5)$ & $13(12.3)$ & $22(20.9)$ & 0.83 \\
Exposición de intestino & No & $32(30.4)$ & $51(48.5)$ & $83(79.0)$ & \\
& Sí & $1(0.9)$ & $9(8.5)$ & $10(9.5)$ & 0.04
\end{tabular}

cuchillo fue el arma blanca más utilizada, en un $8.5 \%$, porcentaje menor al del estudio publicado por Clarke en 2008, en Glasgow, Escocia, de $31.9 \% .{ }^{10}$ El epigastrio fue el área abdominal con mayor frecuencia de lesiones, 20.9\%. En el 98\% de los pacientes se encontró una única herida; con respecto a esto, Pinedo informó $22.4 \%$ de lesiones en el epigastrio y $60 \%$ con una sola herida. ${ }^{1}$ El horario en el que ocurrieron las lesiones podría estar relacionado con el estado etílico en que se encontraban los pacientes en ese periodo (según el INEGI, ${ }^{11}$ Yucatán es el estado que ocupa el primer lugar en alcoholismo a nivel nacional): ante la imposibilidad de resolver los problemas de manera verbal, se recurre a la agresión física, utilizando para ello los objetos punzocortantes más cercanos, entre ellos el cuchillo, lo que refleja la mayor frecuencia de trauma por dicha arma blanca. Debido a que nuestros datos se recabaron de expedientes y no al momento de la atención del paciente, en varios casos no se especificó el arma utilizada. En cuanto al área afectada, esta podría deberse a la dinámica del trauma y la localización central del epigastrio, lo que contribuye a que sea blanco vulnerable de agresiones externas. Se encontró diferencia significativa entre la población de la ciudad de Mérida y la ocurrida en el interior del estado ( $p$ $=0.05$ ). Esto podría estar condicionado por la violencia en la que conviven los estratos de la población más desfavorecidos, ya que esta es una manera de relacionarse con los otros desde edades tempranas.
El tiempo de resolución quirúrgica tras el ingreso fue de nueve horas, superior al reportado por Pinedo y Demetriades, de dos y 0.30 horas, respectivamente. ${ }^{1,12}$ Con respecto a la variable ingreso y resolución quirúrgica, esta podría explicarse por las diferencias de atención entre las áreas urbana y rural: mientras que en el área urbana los tiempos de respuesta y atención son más cortos, en la rural son prolongados, aunados a la carencia de un protocolo intrahospitalario específico para el TPA. En cuanto a las vísceras huecas, el intestino delgado fue el más lesionado, y le siguió en frecuencia el intestino grueso; datos similares han encontrado autores como Pinedo, a nivel nacional, ${ }^{1}$ y Paydar y Lapu, a nivel internacional., ${ }^{5,6}$ En relación con los órganos sólidos, el hígado resultó con mayor frecuencia de daños, seguido por el riñón y el bazo; estos datos son similares a los informados por Demetriades en 2006 en un centro de trauma de primer nivel en los Ángeles, California. ${ }^{12}$ Es importante mencionar que se utilizaron puntos de sutura (cierre) en $0.9 \%$, en comparación con el $34.2 \%$ señalado por Demetriades; observamos un 100\% de esplenectomías y nefrectomías, cifras superiores a las indicadas por el mismo autor, de $73.9 \%$ y $33 \%$, respectivamente. En nuestros resultados, el $5.7 \%$ de las lesiones a órganos sólidos no ameritaron tratamiento quirúrgico específico, lo cual, si bien es coherente, son valores menores a lo encontrado por Demetriades, quien señala que hasta $23.7 \%$ de las lesiones a órganos sólidos no presentaban sangrado activo al momento de la cirugía. ${ }^{12}$ 
La estancia hospitalaria promedio a nivel nacional va desde cuatro hasta 13.16 días. En Yucatán, la estancia promedio registrada ha sido de 10 días; en nuestro estudio, el ingreso promedio fue de seis días, acorde a lo señalado tanto en la literatura nacional como internacional. ${ }^{1,7,9,12} \mathrm{La}$ mortalidad observada en nuestra investigación fue de $0 \%$, en relación con $2 \%$ según Pinedo, ${ }^{1}$ considerando que en nuestro trabajo únicamente se incluyeron pacientes con heridas penetrantes en el abdomen anterior, sin lesiones en otras partes del cuerpo, y que resultaron sin patologías concomitantes que aumentaran la mortalidad.

Encontramos una frecuencia de laparotomías no terapéuticas en un 39\%, similar a lo informado por Nabeel en Gran Bretaña en 2011, de $34.9 \%$, y Castillo, de 35\%; y menor a la observada por Pinedo, de $46.94 \%$, y Demetriades, de $42 \% .1,7,12,13$ Desde 1960, Shaftan preconizó que las laparotomías obligatorias en trauma penetrante de abdomen resultan negativas en un tercio. ${ }^{4}$

En cuanto a los signos vitales, éstos se registraron al ingreso y después de la reanimación básica en el Servicio de Urgencias. Al analizar la variable tensión arterial (TA), no hallamos diferencia significativa entre ambos grupos. Hasler, en 2011, en su estudio que incluyó 47,927 pacientes, comunicó que aquéllos con TA sistólica entre 130-139 mmHg tuvieron una mortalidad de 2.4\%; los que registraron una TA sistólica mayor de $200 \mathrm{mmHg}$, de $7.3 \%$, y en quienes se encontró una TA sistólica menor a $70 \mathrm{mmHg}$, la mortalidad fue de $33.5 \%$; concluyó que la tasa de mortalidad aumenta mientras más disminuya la TA sistólica en pacientes con trauma, por debajo de 130-139 mmHg. ${ }^{14}$ Sin embargo, estos datos difieren con nuestros hallazgos; esto a pesar de tomar como valor de corte en nuestro estudio la TA sistólica menor a $100 \mathrm{mmHg}$ como valor de hipotensión, cuando en la literatura reciente se recomienda tomar puntos de corte mayores, con la finalidad de evitar incluir pacientes con hemorragia activa $y / 0$ inestables. ${ }^{13}$

Con respecto a la frecuencia cardiaca, observamos una diferencia significativa $(p=0.01)$ entre el resultado de la laparotomía terapéutica y la no terapéutica al momento de su ingreso al Servicio de Urgencias. En nuestro estudio, las frecuencias cardiacas se midieron con la palpación del pulso. Como valor de corte tomamos 60-80 latidos por minuto. De acuerdo al
Colegio Americano de Cirujanos, la frecuencia cardiaca se altera en pacientes con choque hipovolémico grado II, con pérdidas de 15 a $30 \%$ del volumen sanguíneo, que representan alrededor de 750 a 1,500 ml de sangre. ${ }^{15}$

En relación con la frecuencia respiratoria, notamos al ingreso una diferencia significativa $(p=0.03)$ en cuanto a las laparotomías terapéuticas. Tomamos como valor de corte una frecuencia de 10-14 respiraciones por minuto. De acuerdo al Colegio Americano de Cirujanos, la frecuencia respiratoria se altera en pacientes con choque hipovolémico grado I, con pérdidas de hasta $15 \%$ del volumen sanguíneo circulante, que representa hasta $750 \mathrm{ml}^{15}$

La irritación abdominal estuvo presente en un $13.3 \%$ versus $67.10 \%$, con significancia estadística ( $p=0.0001)$; la ausencia de peristalsis fue de $7.6 \%$ versus $45.57 \%$, datos según Pinedo. ${ }^{1}$ Con respecto a la exploración física, Clarke observó que casi la totalidad de los pacientes con datos de peritonitis resultaban en laparotomías terapéuticas, por lo que resalta la importancia de laparotomías exploradoras ante la presencia de datos de irritación abdominal. ${ }^{10}$

La exposición de epiplón fue de $20.9 \%$ versus $15.19 \%$, y de asas intestinales, $9.5 \%$ versus $7.6 \%$, según datos cotejados con Pinedo. ${ }^{1}$ En los pacientes con exposición de epiplón y resultado de laparotomía terapéutica, encontramos una frecuencia de $12.3 \%$ versus $58.3 \%$; en aquéllos con exposición de asas intestinales y laparotomía terapéutica hallamos $8.5 \%$ versus $66.6 \%$, datos publicados por Pinedo. ${ }^{1}$ En nuestro estudio observamos diferencias significativas $(p=0.04)$ ante la exposición de asas intestinales y el resultado de laparotomías, considerando que la ausencia de exposición de asas intestinales no descarta el resultado no terapéutico de las laparotomías. Autores como Lapu, ${ }^{6}$ Díaz, ${ }^{8}$ Clarke $^{10}$ y Demetriades ${ }^{12}$ informaron que la ausencia de irritación abdominal con exposición de epiplón o asas intestinales no es criterio absoluto para cirugía obligatoria. La exposición de epiplón o asas intestinales puede ser considerada una indicación relativa, ya que, como indica Ertekin, ${ }^{16}$ la laparotomía es necesaria en $77 \%$ de las evisceraciones de epiplón y $80 \%$ de las veces con exposición de intestino.

Lapu basó su trabajo únicamente en la exploración física y radiografías simples de abdomen; manejó de manera conservadora al 
$60 \%$ de los pacientes. Posteriormente, el $17 \%$ presentó datos de compromiso abdominal y/o hemodinámico que ameritaron intervención quirúrgica. ${ }^{6}$ Investigaciones como la de Lapu basaron sus decisiones tanto del manejo quirúrgico como del conservador en la presencia de datos de inestabilidad hemodinámica; sin embargo, Ertekin, en 2005, basó su decisión posterior a la reanimación de líquidos, exámenes físicos seriados y estudios de imagen complementarios. ${ }^{16}$

Es importante destacar que el error que se debe evitar ante el manejo del trauma penetrante de abdomen es tratar de implementar protocolos de alta complejidad en instituciones de baja complejidad, como describe Brahin. ${ }^{17}$ Con respecto a los signos clínicos obtenidos mediante exploraciones abdominales seriadas, Sanei ${ }^{3}$ observó una sensibilidad y especificidad de $87.3 \%$ y $93.5 \%$, respectivamente, y Clarke, en su informe sobre el impacto de las recomendaciones para el manejo del trauma penetrante, dio a conocer una especificidad de $99 \%{ }^{10}$

\section{CONCLUSIÓN}

En Yucatán, el trauma penetrante de abdomen anterior es más frecuente en pacientes masculinos, solteros, en la cuarta década de la vida, que habitan en el interior del estado. Como causa más frecuente está la agresión por terceras personas asociada con el consumo de alcohol, con resultado de laparotomías no terapéuticas en 39\%.

En pacientes que presentan heridas en el abdomen anterior, en condiciones estables y asintomáticos, no se puede descartar un manejo quirúrgico; sin embargo, la inestabilidad hemodinámica y la irritación peritoneal son factores para considerar un procedimiento terapéutico.

\section{REFERENCIAS}

1. Pinedo OJ, Guevara TL, Sánchez AM. Trauma abdominal penetrante. Cir Ciruj. 2006; 74: 431-442.

2. Oyo-Ita A, Ugare UG, Ikpeme IA. Surgical versus nonsurgical management of abdominal injury. Cochrane Database Syst Rev. 2012; 11: 1-20.

3. Sanei B, Mahmoudieh M, Talebzadeh H, Shahabi S, Aghaei Z. Do patients with penetrating abdominal stab wounds require laparotomy? Arch Trauma Res. 2013; 2: $21-25$.

4. Shaftan GW. Indications for operation in abdominal trauma. Am J Surg 1960; 99: 657-664.

5. Paydar S, Salahi R, Izadifard F, Jaafari Z, Abasi H, Eshraghian A, et al. Comparison of conservative management and laparotomy in the management of stable patients with abdominal stab wound. Am J Emerg Med. 2012; 30: 1146-1151.

6. Lapu K, Mathew M, Gende G, Kevau I. Selective surgical management of penetrating anterior abdominal wounds at the Angau Memorial Hospital: a prospective study. P N G Med J. 2011; 54: 48-52.

7. Castillo JM, Enríquez DL, Herrera RF, Díaz RJ. Laparotomía no-terapéutica en trauma penetrante abdominal y su relación con el estado de intoxicación por alcohol y drogas. Cir Gen. 2011; 33: 232-235.

8. Díaz-Rosales JD, Enríquez-Domínguez L, CastilloMoreno J, Herrera-Ramírez F. Leucocitosis prequirúrgica como factor predictivo de lesión a órgano intrabdominal en trauma penetrante abdominal. Cir Cir. 2012; 80: 516-522.

9. Romero-Peñalosa R. Trauma abdominal en el Hospital Escuela O’Horán. (Especialidad en Cirugía). Universidad Autónoma de Yucatán; 1989.

10. Clarke S, Stearns A, Payne C, McKay AJ. The impact of published recommendations on the management of penetrating abdominal injury. Br J Surg. 2008; 95 : 515-521.

11. Instituto Nacional de Estadística y Geografía. Análisis sobre las principales adicciones en México (alcoholismo, drogadicción y tabaquismo) y sus respectivos programas de prevención. México, DF; 2013. pp. 96-115.

12. Demetriades D, Hadjizacharia P, Constantinou C, Brown C, Inaba K, Rhee P, et al. Selective nonoperative management of penetrating abdominal solid organ injuries. Ann Surg. 2006; 244: 620-628.

13. Nabeel S, Rushing A, Haut E, Kisat M, Villegas C, Chi $A$, et al. Outcome of selective non-operative management of penetrating abdominal injuries from the North American National Trauma Database. Br J Surg. 2011; 99: 155-165.

14. Hasler RM, Nueschc E, Jüni P, Bouamra O, Exadaktylos A, Leckya F. Systolic blood pressure below 110 $\mathrm{mmHg}$ is associated with increased mortality in blunt major trauma patients: multicentre cohort study. Resuscitation. 2011; 82: 1202-1207.

15. Colegio Americano de Cirujanos, Comité de Trauma. Programa avanzado de apoyo vital en trauma para médicos, 8. ${ }^{a}$ edición. American College of Surgeons; 2008: 59-88. ISBN: 978-1-880696-37-8.

16. Ertekin C, Yanar H, Taviloglu K, Guloglu R, Alimoglu O. Unnecessary laparotomy by using physical examination and different diagnostic modalities for penetrating abdominal stab wounds. Emerg Med J. 2005; 22: 790-794.

17. Brahin A, Pero D. Tratamiento no quirúrgico de los traumatismos abdominales. In: Sociedad Argentina de Medicina y Cirugía de Trauma. Trauma, Prioridades. Buenos Aires, Argentina: Panamericana; 2002. pp. 310-113. ISBN: 950-06-20448.
Correspondencia:
Sergio Isidro Gamboa Hoil
Calle 61 Núm. 680J, entre 98 y 100,
Col. Francisco I Madero, 97000,
Mérida, Yucatán, México.
Cel. 0449991899848
E-mail:sergio_gamboa@@hotmail.com 